Original Article

\title{
Effects of the centrifugal contraction exercise of the rectus abdominis accompanied with the isometric exercise of the transversus abdominis on pain and balance of lower back pain patients
}

\author{
JUNG-Ho LEE ${ }^{1)}$ \\ 1) Department of Physical Therapy, Kyungdong University: Bongpo-ri, Toseong-myeon, Goseong-gun, \\ Gangwon-do, Republic of Korea
}

\begin{abstract}
Purpose] The current study attempted to examine the effects of centrifugal contraction exercise of the rectus abdominis during the isometric contraction of the transversus abdominis on pain and balance of patients with chronic back pain. [Subjects and Methods] The subjects included thirty patients who were diagnosed with back pain due to causes other than neurological by a specialist after conducting a medical precision diagnosis. The experiment was performed after evenly dividing the subjects into two groups, namely, control and experimental, using a random method. This study measured pain by using the visual analog scale. To determine the patients' balancing ability using the Multifunktionale trainsgerate balance measurer. [Results] All groups showed a statistically significant within-group difference in visual analog scale and balance change. However, no significant between-group difference was observed in the comparison of therapy effects. [Conclusion] Strengthening the rectus abdominis can be an important factor for increasing the stability and reducing the pain of the lumbar area among patients with back pain. Key words: LBP, Rectus abdominis, Transversus abdominis
\end{abstract}

(This article was submitted Apr. 25, 2017, and was accepted May 24, 2017)

\section{INTRODUCTION}

More than $60-90 \%$ of the entire population experience back pain at least once in their lifetime. Although $90 \%$ patients recover regardless of the cause, half of the remaining $10 \%$ develop chronic back pain with continuous pain ${ }^{1)}$. Patients with chronic back pain that has a high recurrence rate avoids physical activity to prevent pain. This decrease in physical activity becomes a cause of histological and structural changes in the muscles surrounding the spine. Consequently, muscle size decreases and the muscles shrink and become painful, thus forming a vicious cycle ${ }^{2}$.

Problems caused by chronic back pain seriously affect physical function in a comprehensive and complicated manner. In general, patients with back pain not only have weak and imbalanced deep muscles in the lower back compared with normal people but also diminished ability to perceive posture or location of the body because of a functional decrease in proprioception. This condition causes problems in spinal column stability and induces habitual back pain ${ }^{3)}$.

In the past, conservative physiotherapy aimed at pain reduction mainly involved electronic therapy, thermal therapy, and traction therapy. Currently, exercise therapy by training the muscles in the abdominal and lower back areas are broadly implemented. Regular exercise strengthens ligaments, bones, tendons, and muscles and provides nutrition to multiple joints and cartilage to improve motor control ability ${ }^{4}$. It also improves patients' functioning by reducing depression, stress, and chronic muscle pain and by giving mental stability. Exercise therapy can be used as a primary therapy scheme for recovering the motor controllability of the muscles of patients with back pain lost due to damage in the deep muscles, such as the pelvis

Corresponding author. Jung-Ho Lee (E-mail: ljhcivapt@naver.com)

(C2017 The Society of Physical Therapy Science. Published by IPEC Inc.

(c) (1) $\odot$ This is an open-access article distributed under the terms of the Creative Commons Attribution Non-Commercial No DerivaBY NC ND tives (by-nc-nd) License. (CC-BY-NC-ND 4.0: http://creativecommons.org/licenses/by-nc-nd/4.0/) 
floor muscle and transversus abdominis ${ }^{5}$.

Balance largely affects the performance of almost all daily activities. It is the ability to maintain the balanced state of the body, which requires posture stability for posture maintenance and voluntary movement as well as balancing ability for physical reaction to external changes ${ }^{6}$. Damage in the lumbar area causes a balance disorder because two fundamental physiological mechanisms are blocked. First, impediment of the somesthetic sense can develop because of changes in proprioception. Second, diminished muscle strength, motor coordination, and somesthetic sense can deteriorate motor reaction ${ }^{7)}$.

Patients with chronic pain experience restrictions in daily life, such the inability to work because of damaged balancing ability. Patients with back pain cannot properly perform static balancing and show different weight distributions compared with normal people. They use different exercise strategies from normal people because of their injury, which is related to pain, sensory motor nerve system, ligaments, and motor system tissues ${ }^{8}$. A prevous study was conducted to examine the effect of classical Pilates exercises on the EMG of abdominal muscles in 32 subjects. In this study, criss-cross exercise had higher abdominal muscle activity than other exercises ${ }^{9}$.

Currently, the cause of chronic back pain and its therapy is being examined from diverse aspects. However, most studies use lumbar stabilizing exercise, which is mainly afferent contraction. Studies investigating the effects of contraction of the transversus abdominis and the rectus abdominis on pain and balance are scarce. The current study attempted to examine the effects of centrifugal contraction exercise of the rectus abdominis during the isometric contraction of the transversus abdominis on pain and balance of patients with chronic back pain.

\section{SUBJECTS AND METHODS}

The subjects included thirty patients who were diagnosed with back pain due to causes other than neurological by a specialist after conducting a medical precision diagnosis (Computed Tomography or Magnetic Resonance Imaging). The experiment was performed after evenly dividing the subjects into two groups, namely, control and experimental, using a random method. The control group performed a lumbar stabilizing exercise after receiving general physiotherapy (10 min hot pack, 5 min ultrasonic therapy, and 20 min Transcutaneous Electrical Nerve Stimulation). The experimental group performed a centrifugal contraction exercise of the rectus abdominis during the isometric contraction of the transversus abdominis after receiving general physiotherapy.

Patients who were receiving medicinal therapy such as painkillers, those with vestibular organ impairment or neurological impairment, those who experienced dizziness or unexpected falling in the last six months, and those with brain disease were excluded from the research. Exercise according to the intervention method was implemented 18 times at three times a week for six weeks.

We sufficiently explained the purpose and method of this study to all the research subjects before the experiment. The participants were provided a written informed consent form in accordance with the ethical standards of the Declaration of Helsinki.

This study measured pain by using the visual analog scale (VAS). VAS was devised to quantify visually the level of pain, and it is an indicator that can be easily used clinically. One end of a $10 \mathrm{~cm}$ straight line is marked with "score 0 of absolutely no pain," and the other end of the line is marked with "score 10 of unbearable pain." The patients were instructed to mark their level of pain from 0 to 10 .

To determine the patients' balancing ability, the front-and-rear balance and the left-and-right balance of the center of weight on an unstable supporting ground were measured using the MFT balance measurer. The measurement results were expressed as a percentage of the latency time of the center of weight that was recorded on target divided by five against the total measurement time. Through the software computation, the time record was converted to the highest score of balancing ability of 0 to the lowest score of 5 . The two groups received exercise treatment for six weeks. Evaluation was implemented before the experiment and after the six-week experiment. The evaluation and treatment of this study were performed by a physical therapist who had a clinical experience of 10 years and orthopedic physiotherapist for more than 250 hours.

In this study, bridge exercise was implemented as a lumbar stabilizing exercise. In a supine posture with both hands comfortably placed on the ground, the patients bended both knees and lifted their pelvis to maintain a neutral posture of the lumbar area for $10 \mathrm{~s}$, followed by a $3 \mathrm{~s}$ resting time. One time of exercise was held for $13 \mathrm{~s}$. Lumbar stabilizing exercise was implemented 10 times after general physiotherapy, followed by a 3 min resting time. One set consisted of 10 times, and three sets were implemented after general physiotherapy.

During the isometric contraction of the transversus abdominis, patients began the centrifugal contraction of the rectus abdominis in a supine posture with a $90^{\circ}$ flexion of the hip joint and a $90^{\circ}$ flexion of the knee joint. Before performing the centrifugal contraction of the rectus abdominis, the patients were told to perform the isometric contraction of the transversus abdominis first. After the patients were instructed about the isometric contraction of the transversus abdominis, the therapist put his/her hands on the patients' Achilles tendon of both sides and placed the patients' legs in the starting position for the exercise. Through the verbal indication of "slowly lower your legs" from the therapist, the patients lowered both legs to the ground while maintaining the knee angle at a $90^{\circ}$ until the hip joint became a $45^{\circ}$ flexion. The centrifugal contraction exercise of the rectus abdominis was implemented 10 times during the isometric contraction of the transversus abdominis, followed by a 3 min resting time. One set consisted of 10 times, and three sets were implemented after general physiotherapy. 
Table 1. Comparison of VAS between groups (Mean $\pm \mathrm{SD})$

\begin{tabular}{lcc}
\hline & $\begin{array}{c}\text { Experimental group } \\
(\mathrm{n}=15)\end{array}$ & $\begin{array}{c}\text { Control group } \\
(\mathrm{n}=15)\end{array}$ \\
\hline Pre-test & $5.65 \pm 1.68$ & $5.82 \pm 1.86$ \\
Post-test & $2.34 \pm 1.08^{*}$ & $2.91 \pm 1.26^{*}$ \\
\hline
\end{tabular}

$\mathrm{p}<0.05, *$ within group
Table 2. Comparison of balance between groups (Mean \pm SD)

\begin{tabular}{lcc}
\hline & $\begin{array}{c}\text { Experimental group } \\
(\mathrm{n}=15)\end{array}$ & $\begin{array}{c}\text { Control group } \\
(\mathrm{n}=15)\end{array}$ \\
\hline Pre-test & $3.55 \pm 0.15$ & $3.87 \pm 0.24$ \\
Post-test & $1.28 \pm 0.28^{*}$ & $1.56 \pm 0.32 *$ \\
\hline $\mathrm{p}<0.05,{ }^{*}$ within group &
\end{tabular}

SPSS 20.0 for Windows was used for the data analysis. We used the paired t-test to compare the difference in pain and balance before and after the experiment. We used the independent t-test to compare the between-group difference in pain and balance. All data values were expressed in mean \pm standard deviation. The significant level was set to $\alpha<0.05$.

\section{RESULTS}

All groups showed a statistically significant within-group difference in VAS change $(\mathrm{p}<0.05)$. However, no significant between-group difference was observed in the comparison of therapy effects ( $p>0.05)$ (Table 1). In this study, a within-group change in balance showed a statistically significant difference in all groups $(p<0.05)$. However, no significant between-group difference was observed in the between-group comparison ( $p>0.05$ ) (Table 2).

\section{DISCUSSION}

Chronic back pain causes inconvenience in daily life because of the limited range of motion of joint, pain, and decreased balance it entails. Chronic back pain is caused by a combination of structural joint problems and functional muscle problems. The early symptom of back pain develops at the center of the lumbosacral area. In severe cases, changing posture is even difficult because of the pain. Sometimes this pain is limited to referred pain in the sacroiliac joints, bottom, groin, or femoral region. However, it is typically accompanied with radiating pain, which progresses from under the knee toward the tip of the toe as a nerve root is stimulated ${ }^{10)}$.

Balance refers to the ability to maintain the center of gravity of the body with supporting ground with minimal sway. Interaction between the factors in the central and peripheral areas is essential for maintaining balance. The peripheral factors consist of the somesthetic sense, which provides information on tension, extension, and pain in the joint, muscle, ligament, and joint location, as well as the vestibular system, which provides information on environmental change ${ }^{11)}$. The central factors integrate the information received from the peripheral factors, and the most appropriate muscle reaction for body trunk location and posture control are chosen. Patients with back pain require an additional program that can enhance balance, as their balancing ability is diminished compared with normal people. The dynamic stability exercise excites the motor organ in the brain by stimulating proprioception to maximize the balance sense and ability to maintain it, consequently improving the balancing ability of patients with chronic back pain ${ }^{12}$.

The lumbar stabilizing exercise stabilizes the spinal column by inducing the exercise of the deep muscles but without the contracting the shallow muscles in the lumbar area as much as possible. If the stabilizing exercise is successful, back pain will be prevented, as the unstable sway in the deep muscle will disappear even if large shallow muscles contract. The lumbar stabilizing exercise has been recently reported to be effective in improving the function of the proprioceptive sense, the ability to perceive correct posture, the left-and-right imbalance, and the dynamic balancing ability of patients with chronic back pain ${ }^{13)}$. Shin implemented the lumbar stabilization exercise to 20 patients with idiopathic scoliosis and reported that their balancing ability measured by total sway distance and distance velocity improved ${ }^{14}$.

This study applied a six-week exercise therapy scheme to patients with chronic back pain to investigate the effect of the centrifugal contraction of the rectus abdominis on pain and balance during the lumbar stabilizing exercise and the isometric contraction of the transversus abdominis. According to the study results, all groups showed decreased pain and improved balance. No significant difference was found in therapeutic effects according to the exercise method. However, the group that performed the centrifugal exercise of the rectus abdominis during the isometric contraction of the transversus abdominis showed a slightly larger change than the group that performed the lumbar stabilizing exercise. These results are due to the strengthening of the rectus abdominis muscle, which increases the stability of the pelvis, resulting in decreased pain and improved balance.

This study has several limitations. First, the sample was small and the application duration of the therapy was short. Second, the study included only patients with back pain in the chronic phase. Therefore, applying this therapy to patients with back pain in the acute or subacute phase is difficult. Third, this study considered only the physical aspect of patients with chronic back pain and did not sufficiently account for the psychosocial aspect. In this study, it is difficult to generalize the results of the study because only the VAS and MFT were used to evaluate the study results. We expect future studies to apply a long-term exercise therapy scheme to a larger number of patients experiencing chronic back pain with diverse occurrence duration and to examine the effects. 


\section{REFERENCES}

1) Cooper NA, Scavo KM, Strickland KJ, et al.: Prevalence of gluteus medius weakness in people with chronic low back pain compared to healthy controls. Eur Spine J, 2016, 25: 1258-1265. [Medline] [CrossRef]

2) Hides J, Gilmore C, Stanton W, et al.: Multifidus size and symmetry among chronic LBP and healthy asymptomatic subjects. Man Ther, 2008, 13: 43-49. [Medline] [CrossRef]

3) Lee CW, Hwangbo K, Lee IS: The effects of combination patterns of proprioceptive neuromuscular facilitation and ball exercise on pain and muscle activity of chronic low back pain patients. J Phys Ther Sci, 2014, 26: 93-96. [Medline] [CrossRef]

4) Searle A, Spink M, Ho A, et al.: Exercise interventions for the treatment of chronic low back pain: a systematic review and meta-analysis of randomised controlled trials. Clin Rehabil, 2015, 29: 1155-1167. [Medline] [CrossRef]

5) Leonard JH, Paungmali A, Sitilertpisan P, et al.: Changes in transversus abdominis muscle thickness after lumbo-pelvic core stabilization training among chronic low back pain individuals. Clin Ter, 2015, 166: e312-e316. [Medline]

6) Kim JH, Kim YE, Bae SH, et al.: The effect of the neurac sling exercise on postural balance adjustment and muscular response patterns in chronic low back pain patients. J Phys Ther Sci, 2013, 25: 1015-1019. [Medline] [CrossRef]

7) Čeko M, Shir Y, Ouellet JA, et al.: Partial recovery of abnormal insula and dorsolateral prefrontal connectivity to cognitive networks in chronic low back pain after treatment. Hum Brain Mapp, 2015, 36: 2075-2092. [Medline] [CrossRef]

8) Gatchel RJ, McGeary DD, McGeary CA, et al.: Interdisciplinary chronic pain management: past, present, and future. Am Psychol, 2014, 69: 119-130. [Medline] [CrossRef]

9) Pereira IL, Queiroz B, Loss J, et al.: Trunk muscle EMG during intermediate pilates mat exercises in beginner healthy and chronic low back pain individuals. J Manipulative Physiol Ther, 2017, S0161-4754(17)30055-6. [Medline]

10) Van Buyten JP, Al-Kaisy A, Smet I, et al.: High-frequency spinal cord stimulation for the treatment of chronic back pain patients: results of a prospective multicenter European clinical study. Neuromodulation, 2013, 16: 59-65, discussion 65-66. [Medline] [CrossRef]

11) Frost LR, Brown SH: Muscle activation timing and balance response in chronic lower back pain patients with associated radiculopathy. Clin Biomech (Bristol, Avon), 2016, 32: 124-130. [Medline] [CrossRef]

12) Etemadi Y, Salavati M, Arab AM, et al.: Balance recovery reactions in individuals with recurrent nonspecific low back pain: effect of attention. Gait Posture, 2016, 44: 123-127. [Medline] [CrossRef]

13) Boucher JA, Preuss R, Henry SM, et al.: The effects of an 8-week stabilization exercise program on lumbar movement sense in patients with low back pain. BMC Musculoskelet Disord, 2016, 17: 23. [Medline] [CrossRef]

14) Shin SS, Lee YW, Song CH: Effects of lumbar stabilization exercise on postural sway of patients with adolescent idiopathic scoliosis during quiet sitting. J Phys Ther Sci, 2012, 24: 211-215. [CrossRef] 